\title{
O Uso da Modelagem Paramétrica no Desenvolvimento de Órteses Para Tecnologia Assistiva
}

The Use of Parametric Modeling in the Orthoses Development for Assistive Technology

CATAPAN, Márcio F.; Dr.; Universidade Federal do Paraná

marciocatapan@ufpr.br

ROHRBACHER, Gustavo; Bel. Expressão Gráfica; Universidade Federal do Paraná

rohrbacher.gv@gmail.com

QUEIROZ, F. M. M.; Eng. Mecânico e Mestrando; Universidade Tecnológica Federal do Paraná

mendesdequeiroz@gmail.com

SANTANA, F. E.; Dr.; Instituto Federal de Santa Catarina

fsantana@ifsc.edu.br

\section{Resumo}

O desenvolvimento de produtos personalizados para tecnologia assistiva, como órteses, tem tido lacunas supridas com o uso de novas tecnologias. Esta pesquisa teve como objetivo propor um método para adaptar o conceito Cortex em uma órtese desenvolvida com modelagem paramétrica. Com auxílio de profissionais da saúde, foram listados pontos críticos da superfície e vulnerabilidade física de regiões de uma órtese, de forma a serem utilizadas na modelagem da nova proposta. Foi gerada uma nova modelagem, já parametrizada, fazendo uso do software Rhinoceros em conjunto com o plug-in Grasshopper. Concluída esta etapa, o modelo foi submetido à aproximação de pontos e adição de nervuras em regiões específicas, para dar rigidez ao produto. Características como redução de material, diminuição de peso, facilidade de higienização e customização de aspectos visuais e estruturais, encontradas nos modelos Cortex, também contribuíram para 0 aperfeiçoamento de produtos para tecnologia assistiva.

Palavras Chave: órtese; Cortex; tecnologia assistiva; modelagem paramétrica.

\begin{abstract}
The development of customized assistive technology products, such as orthoses, has been gaps filled with the use of new technologies. The aim of this research was to propose a method to adapt the Cortex concept in a orthosis developed through parametric modeling. Supported by health professionals, we listed critical points of the surface and physical vulnerability of regions of this orthosis to be applied in the new proposal modeling. In this way a new modeling was generated, already parameterized, using the Rhinoceros software together with the Grasshopper plug-in. After, the model was submitted to point approximation and addition of ribs in specific regions, to give rigidity to the product. Features such as material reduction, weight reduction, ease of hygiene and customization of visual and structural aspects, present in the Cortex models, also contributed to the improvement of assistive technology products.
\end{abstract}

Keywords: orthoses; Cortex; assistive technology; parametric modeling. 


\section{Introdução}

O uso da prototipagem rápida vem revolucionando a indústria a cada novo passo, através de modelagens e impressões 3D feitas em softwares CAD e CAM. Esses, por sua vez, podem ser ainda mais versáteis se usados de forma paramétrica, com intuito de atender um maior número de usuários sem um padrão definido. Ou seja, temas como modelagem paramétrica e tecnologia assistiva estão diretamente relacionada a este assunto.

Foggiatto et al (2007) define que a modelagem paramétrica permite gerar modelos com dimensões vinculadas a variáveis, possibilitando que este seja regenerado automaticamente após modificações.

Por definição, Bersch (2013) cita que, Tecnologia Assistiva (TA), é um termo utilizado para identificar todo arsenal de recursos e serviços que proporcionam ou ampliam habilidades funcionais de pessoas com deficiência. Galvão Filho (2009), define que órteses, assim como próteses, são consideradas recursos de TA e se encontram classificadas em Norma Internacional ISO 9999:2002. Por sua vez, não seguem um padrão de usuário fixo, visto que a anatomia de cada paciente se mostra diferente.

Padrini (2006), salienta que as órteses são desenvolvidas com base na necessidade específica de cada paciente e podem ser utilizados para uma gama de fatores em busca da cura do problema, a exemplo das contraturas.

Nesse contexto que está inserido o conceito Cortex, ou "gesso do futuro", que é modelado através de técnica de parametrização e fabricado por manufatura aditiva, é utilizado para imobilização de membros fraturados, assim como os gessos ortopédicos que podem ser caracterizados como órteses. (DEZEEN MAGAZINE, 2013)

Define-se então que o objetivo deste trabalho é aplicar o conceito Cortex em um modelo de órtese estática e personalizada, fazendo uso de modelagem paramétrica. Para cumprir isso, os objetivos específicos são:

- Analisar a viabilidade dos processos de desenvolvimento de produtos, semelhantes ao Cortex, na fabricação de órteses para tecnologia assistiva;

- Propor novo design para as atuais órteses de tecnologia assistiva;

- Analisar se o modelo parametrizado pode agilizar a modelagem de órteses;

- Avaliar se o modelo gerado é mais apropriado ao usuário.

\section{Revisão Bibliográfica}

\subsection{Tecnologias Assistivas (TA)}

Cook e Hussey (1995), definem TA como sendo "uma ampla gama de equipamentos, serviços, estratégias e práticas concebidas e aplicadas para minorar os problemas funcionais encontrados pelos indivíduos com deficiências".

Em conjunto com o ADA - American with Disabilities Act, Sartoretto e Bersch (2014) salientam que os projetos envolvendo TA muitas vezes são de grandezas jurídicas. Isto é, além de garantir direitos a cidadãos portadores de deficiências, provém de base legal para utilização de fundos públicos para compra dos recursos que beneficiários desses direitos necessitam. Bersch 
(2013) já descreveu que os recursos de TA são classificados de acordo com a necessidade funcional a que são destinados.

\section{2 Órteses}

Van Petten et al. (2014, apud Foggiatto, Poier e Fernandes, 2015) afirmam que pessoas com deficiência são usuárias de órteses entre outros dispositivos de tecnologia assistiva, devido as suas habilidades funcionais comprometidas.

Outros autores como Edelstein e Bruckner (2006) e Bersch (2013) definem órteses como sendo sistemas acoplados junto a um segmento do corpo e servem para garantir um melhor posicionamento, estabilização e/ou função. Desta forma, os benefícios terapêuticos das órteses podem ser listados quanto a limitação do movimento, assistência ao movimento, transferência de força e proteção de partes do corpo.

Goia (2012) define as órteses estáticas como aquelas que permitem pouco ou nenhum movimento do membro, não possuem componentes móveis e são utilizadas a fim de imobilização para proteger, reduzir dor, evitar deformidades e manter o alinhamento dos membros. Isso evita deformidades impedindo contraturas pelo posicionamento. Ao tratar da fabricação, o autor ainda as divide em dois grupos, sendo: pré-fabricadas e personalizadas.

Padrini (2006) cita que as órteses pré-fabricadas, ainda que comercializadas em lojas específicas, perdem mercado para as órteses sob medida, devida à dificuldade de adaptação dos pacientes na utilização produtos não personalizados. $O$ autor ainda afirma que toda órtese deve ser única, pois é criada com base na necessidade específica de cada paciente.

\subsection{Tecnologias CAD/CAM e a Manufatura Aditiva no Projeto e Fabricação de Órteses}

Segundo Volpato et al. (2007), as técnicas de manufatura aditiva, consistem em um processo de construção tridimensional que se dá por meio da adição de material. Ao definir qual dos processos atuais é o mais adequado a se utilizar, Medeiros et al. (2014), defende o uso da tecnologia FDM (Fused Deposition Modeling).

O mesmo avanço em sistemas CAD que impulsionou a manufatura aditiva, fez com que estes sistemas de modelagem pudessem trabalhar com formas mais livres e orgânicas, dando existência ao termo denominado Modelagem Paramétrica (MP).

Segundo Speck (2001, apud Foggiatto, Volpato, e Bontorin, 2007), a modelagem sólida paramétrica permite a geração de modelos com dimensões vinculadas à variáveis, permitindo a regeneração automática do modelo após cada modificação, havendo assim ganho de tempo durante as modificações do projeto. Esse contexto é ilustrado na Figura 1.

Segundo Celani e Pupo (2008), o processo de parametrização torna-se otimizado, assim permitindo maior celeridade às fases de projeto e confecção do produto. Barbosa et al. (2012) evidenciam que: a utilização do software de modelagem tridimensional Rhinoceros juntamente com o plug-in Grasshopper - Generative modeling for Rhino - permitiu a construção de um modelo paramétrico tridimensional. Nesse caso, à medida que os valores dos parâmetros estabelecidos no Grasshopper são alterados, o modelo geométrico é automaticamente atualizado. 
Figura 01: Modificações de modelo com uso de parametrização
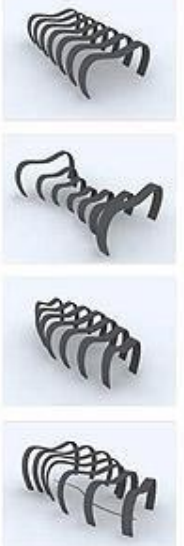
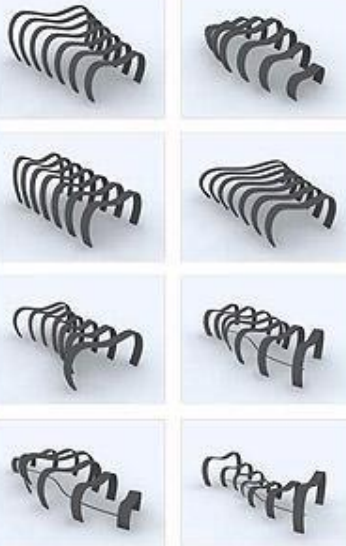
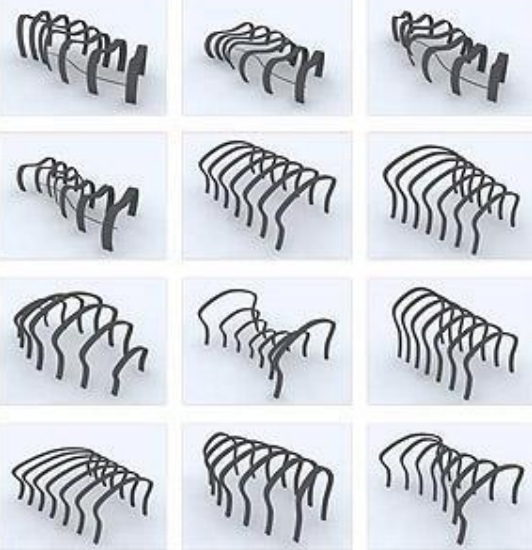

Fonte: Yalinay et al. (2008)

\subsection{Conceito Cortex}

Apresentado por DeZeen Magazine (2013) como sendo um modelo impresso em 3D, de formas livres e complexas que auxiliam na usabilidade do modelo. Suas características positivas em relação ao gesso ortopédico comum é que este, além de leve, ventilável, lavável, higiênico, reciclável e resistente, evita-se coceira e mau cheiro como nos antigos métodos de engessamento.

DeZeen Magazine (2013) esclarece que desenvolvimento do Cortex é realizado em três passos, demonstrado na Figura 2:

- Escaneamento do membro estudado para obter a geometria;

- Uso da geometria escaneada para realização da modelagem através de parâmetros;

- Impressão tridimensional.

Figura 2: Processo de desenvolvimento do Cortex

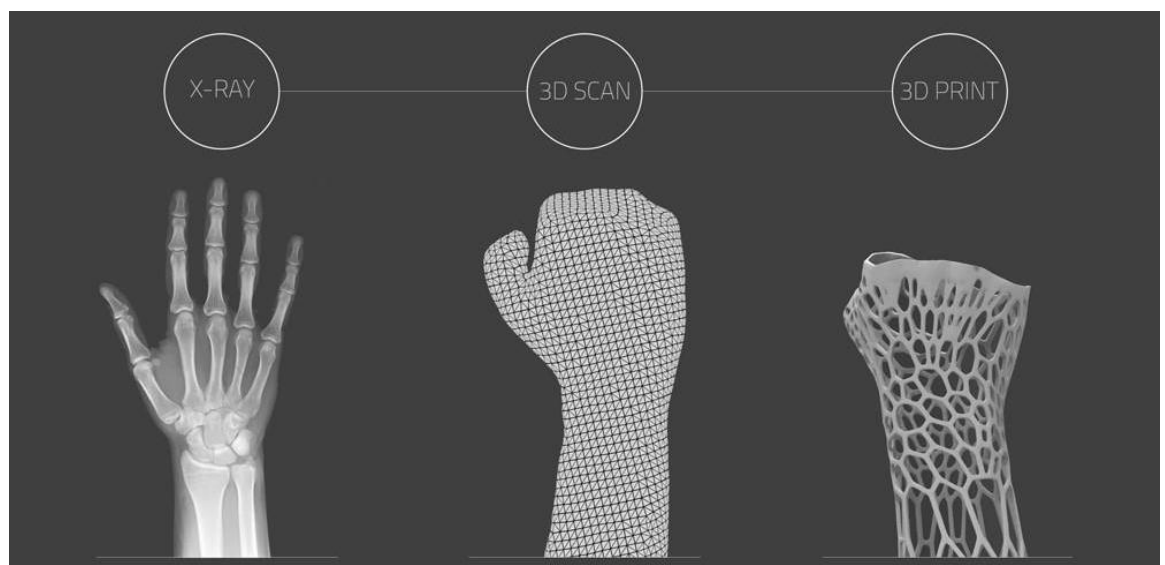

Fonte: Adaptado de Dezeen Magazine (2016)

\section{Materiais e Métodos}

Tendo em vista aprimorar processos de desenvolvimento de órteses para pacientes com paralisia cerebral, Catapan et al. (2017) propõe um método semelhante a Villas Boas et al. (2016). 
O estudo atual se dá para um paciente de 2 anos e meio de idade com deficiência em membro inferior. O caso chamou atenção dos autores devido ao alto grau de contratura do membro afetado em relação a baixa idade do paciente.

Catapan et al (2017) relata ser necessário entrar em contato com profissionais da área da saúde, na ocasião um terapeuta ocupacional e uma fisioterapeuta, em busca de auxílio para posicionar corretamente o membro a ser escaneado. Com a ajuda desses profissionais foi feito o relaxamento da musculatura do paciente para gradualmente posicionar o membro na posição desejada (Figura 3), podendo assim, extrair a geometria esperada do membro afetado.

Figura 3: Relaxamento e avaliação do membro afetado

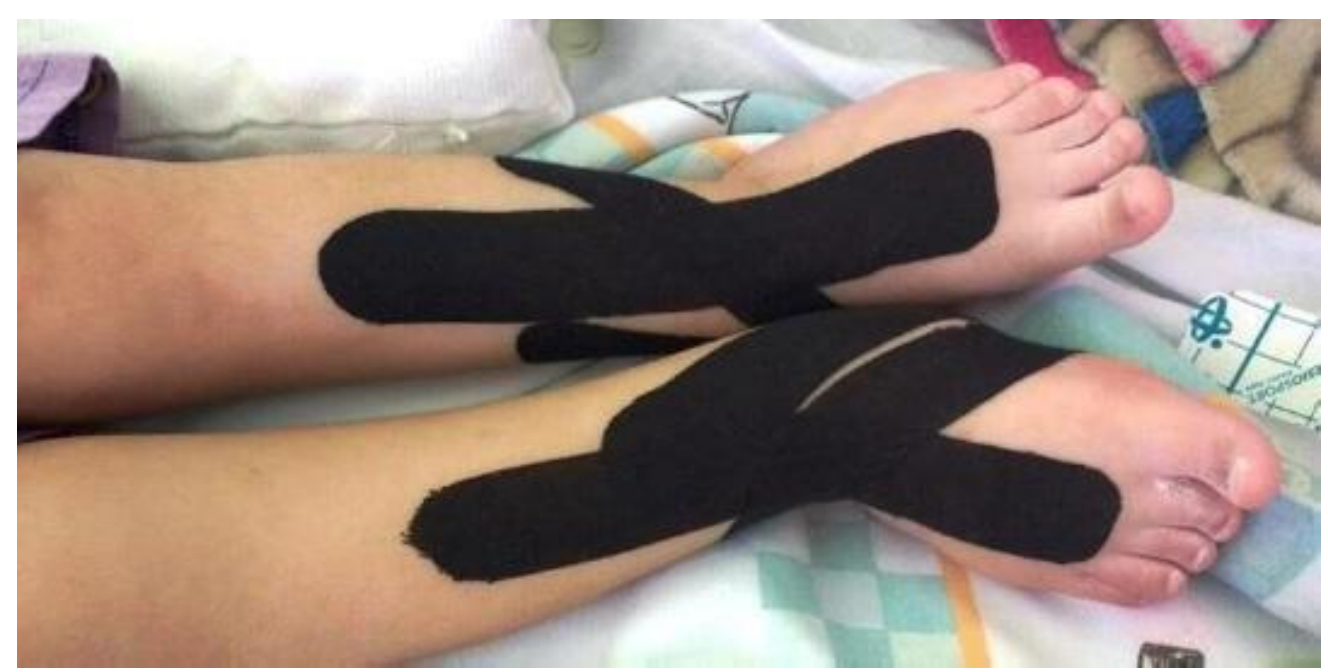

Fonte: Catapan et al. (2017)

Após avaliação do membro afetado (Figura 3), Catapan et al. (2017) utilizam o método de extração da geometria, de forma indireta, por meio de atadura gessada. Em seguida, com o molde de gesso curado, esse foi aberto e submetido ao escaneamento para capturar a geometria do membro.

Após o escaneamento é gerado um arquivo ".stl" e exportado para um software de modelagem com intuito de tratar e suavizar a malha. Após isso, foram inseridas abertura de rasgos para colocação de velcros. Finalizando o modelo foi aplicado um offset de $7 \mathrm{~mm}$ de espessura, deixando a modelagem finalizada para ser impressa (CATAPAN ET AL, 2017).

Após o modelo (.st/) ser tratado e finalizado, este passou pelo processo de manufatura aditiva com o uso de uma impressora 3D Cloner modelo $\mathrm{DH}+$, utilizando ácido poliláctico (PLA). Com a aplicação da órtese no paciente, como demonstrado na Figura 4, é possível notar o membro corrigido com o uso da mesma.

Segundo Catapan et al. (2017), o resultado final da órtese se mostrou satisfatório, mesmo que com alguns ajustes sugeridos através de um questionário respondido pela equipe de saúde formada por terapeutas ocupacionais e Fisioterapeutas. 
Figura 4: Aplicação da órtese no membro afetado

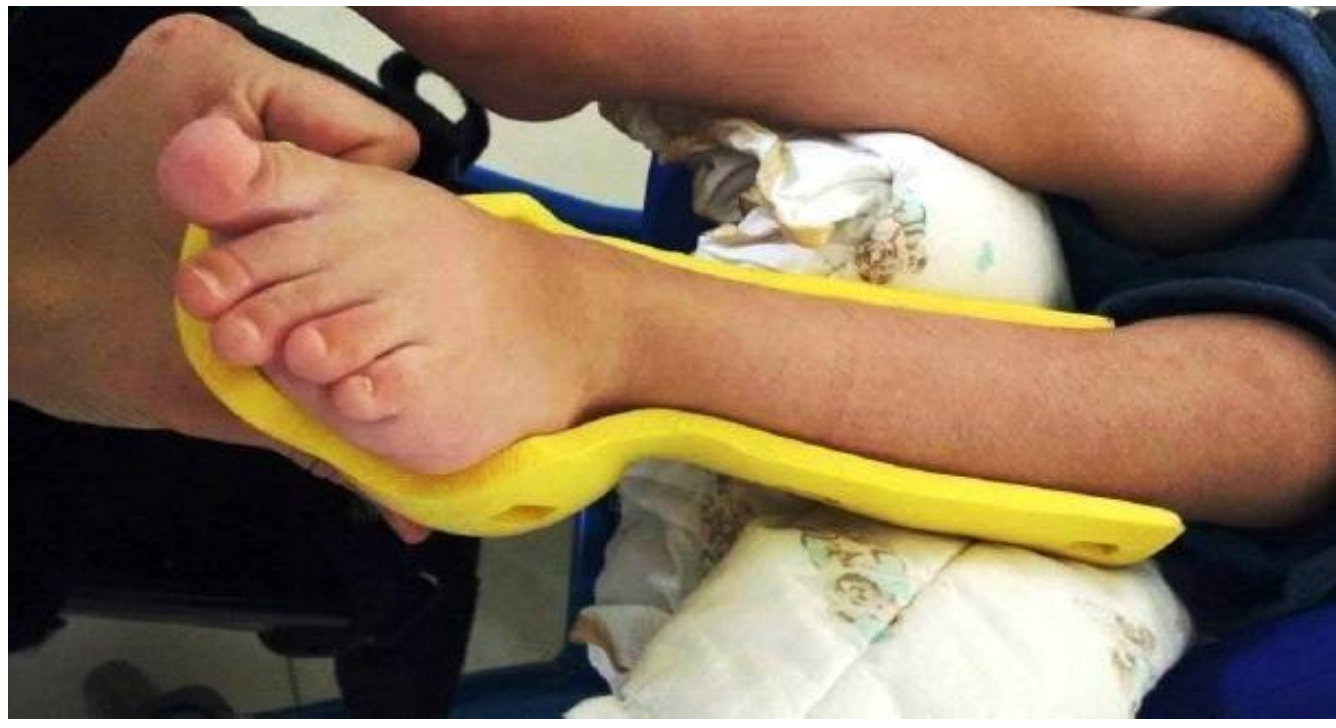

Fonte: Catapan et al. (2017)

Entretanto, independente da maneira que é gerada a órtese, sabe-se que essa forma pode gerar pequenos problemas ao usuário, como suor e até mesmo escaras. Sendo assim, o intuito deste trabalho está no desenvolvido de uma órtese, semelhante a demonstrada até aqui, em conceito Cortex.

\section{Desenvolvimento}

Embora a pesquisa de Catapan et al. (2017) tenha sido finalizada de forma satisfatória. Esse desenvolvimento da modelagem proposta neste trabalho busca apresentar um novo conceito para um desenvolvimento de uma órtese estática parametrizada para ser utilizada nas pesquisas do campo de tecnologia assistiva.

\subsection{Nova proposta para a órtese de Catapan et al (2017)}

Através do modelo tridimensional da órtese customizada, usada por Catapan et al (2017), foi utilizado o software Meshmixer da Autodesk para se trabalhar novamente na malha.

A nova espessura adotada foi de $4,0 \mathrm{~mm}$, utilizada também no estudo de Villas Boas et al. (2016). Ao finalizar a modelagem, a órtese foi fabricada em PLA por meio de FMD utilizando uma impressora ZMORPH 2.0 VX disponibilizada pelo Laboratório de Manufatura e Prototipagem - LAMP do Departamento de Expressão Gráfica - DEGRAF da UFPR - Universidade Federal do Paraná. Conforme apresentado na Figura 5.

O propósito em replicar o modelo tridimensional desenvolvido por Catapan et al (2017) e apresentado na Figura 5, é que agora essa órtese poderá ser melhor estudada pelos autores deste artigo em parceria com os profissionais da área de saúde. Um estudo muito importante e que será descrito a seguir é a análise dos pontos de tensão da órtese. 
Figura 5: Protótipo impresso

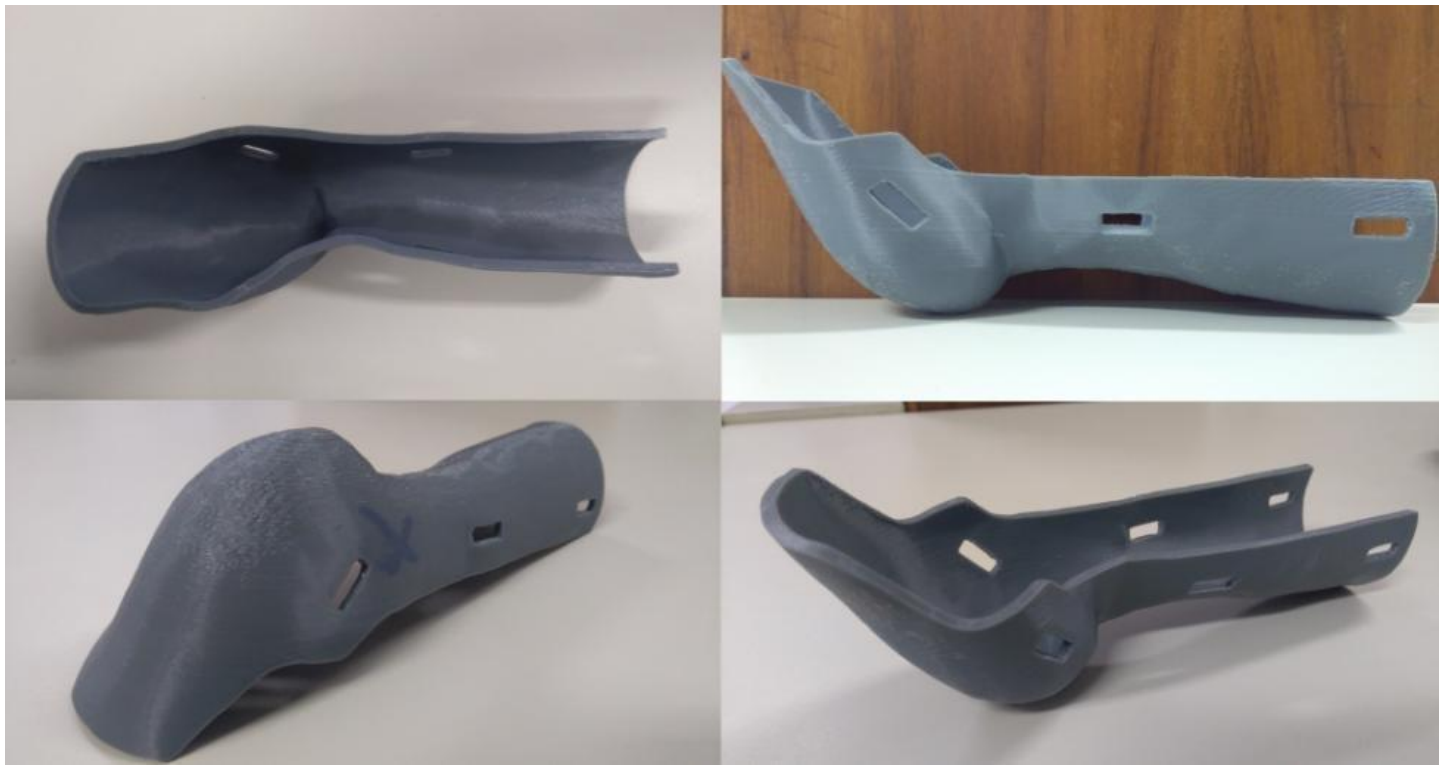

\subsection{Avaliação dos pontos de tensão do protótipo}

Ao avaliar, junto ao terapeuta ocupacional, os pontos de tensão da órtese fabricada, podese aumentar a rigidez de pontos específicos bem como criar pontos de alívio a fim de melhorar o conforto do paciente, conforme mostrado na Figura 6.

Figura 6: Vistas dos pontos de tensão - parte externa do tornozelo
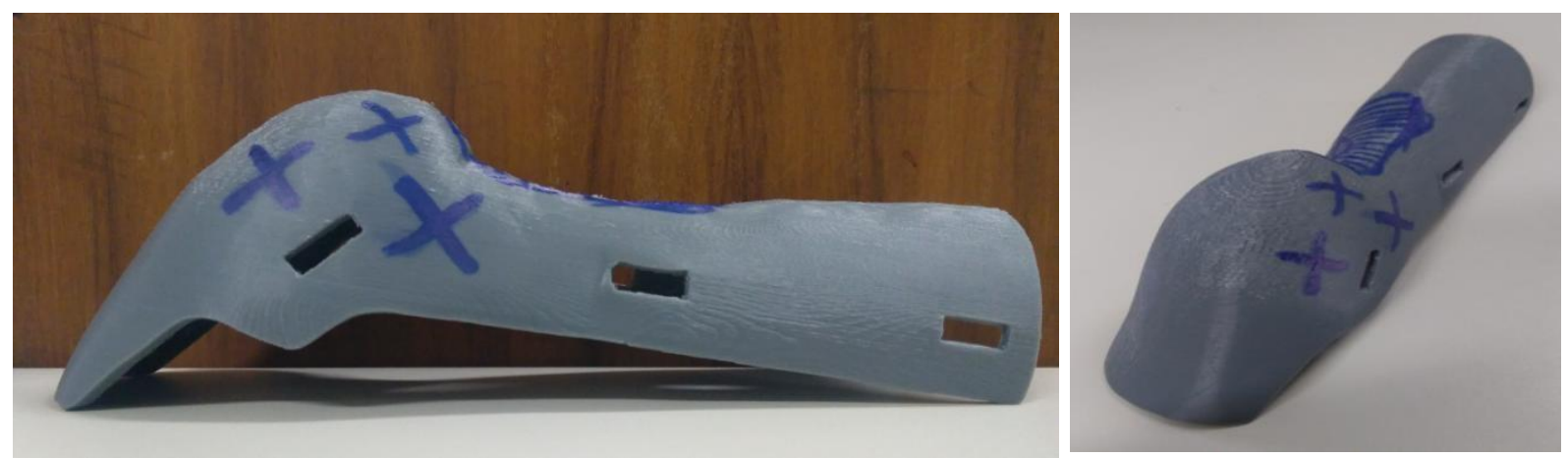

As marcações em " $X$ " apontam regiões onde há maior solicitação mecânica. Essas tensões se dão ao movimento do pé se recolher/atrofiar para o sentido interno da perna, forçando a região oposta, podendo facilitar o rompimento da órtese na região.

As marcações de linhas paralelas indicam as regiões que necessitam um aumento médio de espessura e linhas cruzadas indicam as regiões que necessitam um alto grau de aumento da espessura, conforme demonstrado na Figura 7.

Realizadas as ponderações, com o auxílio do profissional de saúde da área de terapia ocupacional, a respeito da vulnerabilidade física e pontos críticos do modelo desenvolvido por Catapan et al. (2017), este modelo foi utilizado para embasar o desenvolvido da proposta final deste 
trabalho.

Figura 7: Marcação das regiões de forte tensão na órtese
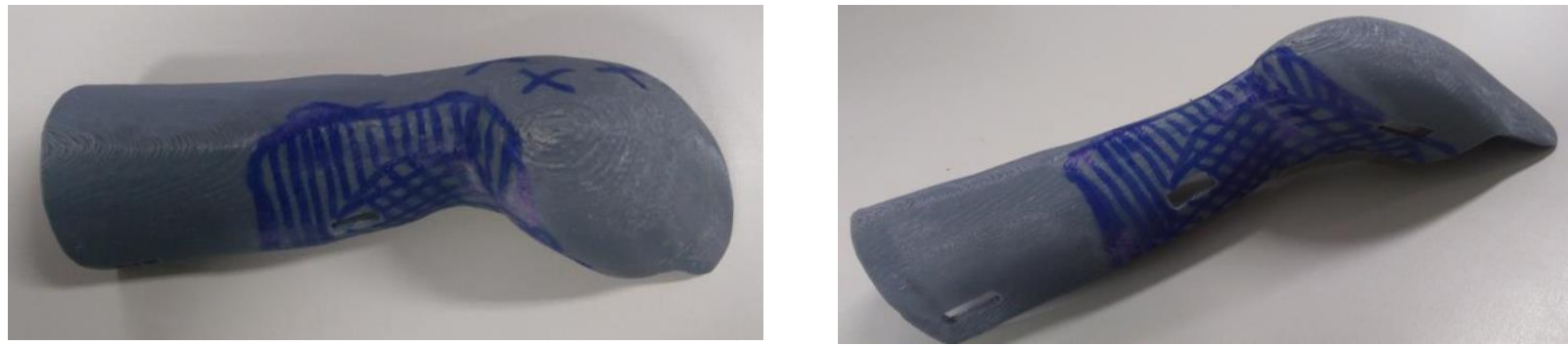

\subsection{PROPOSTA FINAL}

Inicialmente foi aplicada a parametrização no modelo de órtese, apresentado no capítulo anterior, para que posteriormente fossem realizadas as alterações verificadas no item 4.2, obtendose assim o modelo parametrizado de órtese estática.

\subsubsection{Modelo paramétrico}

Com o estudo dos pontos de tensão críticos da órtese finalizado, a mesma é submetida a parametrização do modelo, na qual foi utilizado o software Rhinoceros e o plug-in Grasshopper.

O plug-in Grasshopper é uma ferramenta desenvolvida para trabalhar, exclusivamente em conjunto com o software Rhinoceros, possibilitando que modelagens orgânicas de formas livres sejam criadas a partir de suas ferramentas paramétricas. A modelagem utilizando o plug-in consiste basicamente em vincular ferramentas complementares interligando-as, formando assim uma nuvem de processos construtivos da modelagem. Esta modelagem é definida como paramétrica devido ao vínculo dessas ferramentas com seletores de valores variáveis.

Iniciada a modelagem é essencial criar, através da nuvem de processos construtivos do Grasshopper, um retângulo vinculado a variáveis utilizadas para definir seu tamanho futuramente. Na figura 8 é possível observar que ao definir os comandos de construção do retângulo no plug-in a forma deste é gerada automaticamente na viewpoint do Rhinoceros.

Figura 8: Construção do retângulo pela nuvem do grasshopper

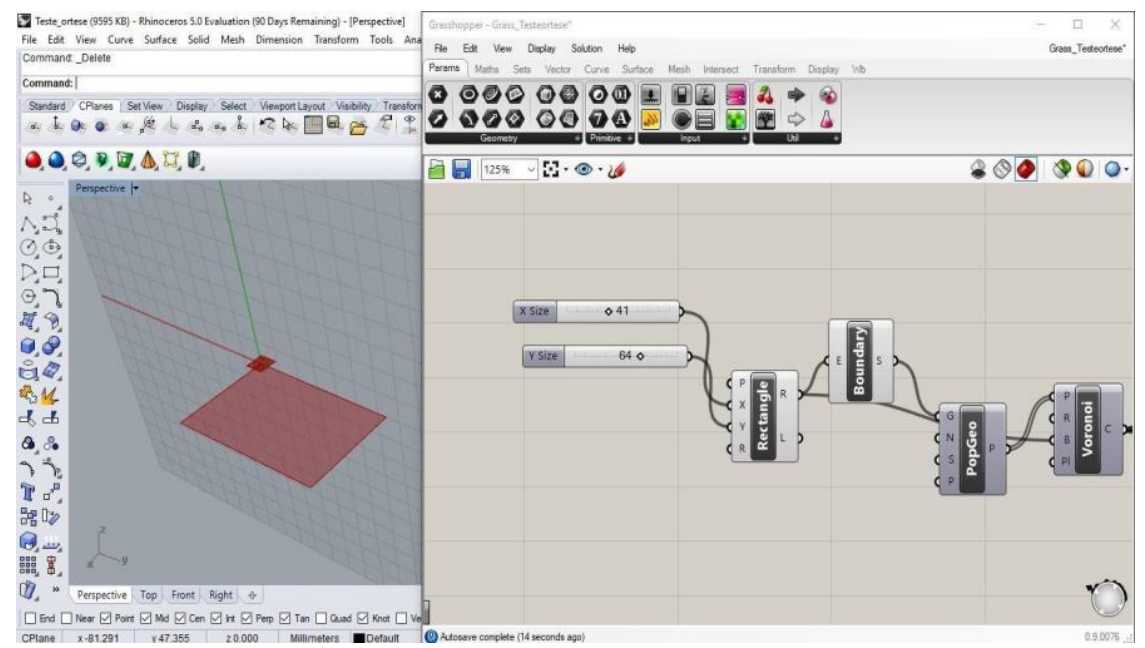


Além destas ferramentas mais comuns, o programa usado ainda possui a ferramenta/modificador Voronoi, primordial para o resultado final da parametrização. Esta ferramenta trabalha com um algoritmo próprio, de formas pré-definidas que são alteradas se vinculadas a outros modificadores. O Voronoi facilita o desenvolvimento da estrutura parametrizada pelo fato das suas predefinições de formas não necessitarem de uma programação específica, agilizando a construção da nuvem de parametrização. Essa definição é possível ser visualizada na Figura 9.

Figura 9: Definição da nuvem de processos construtivos no grasshopper

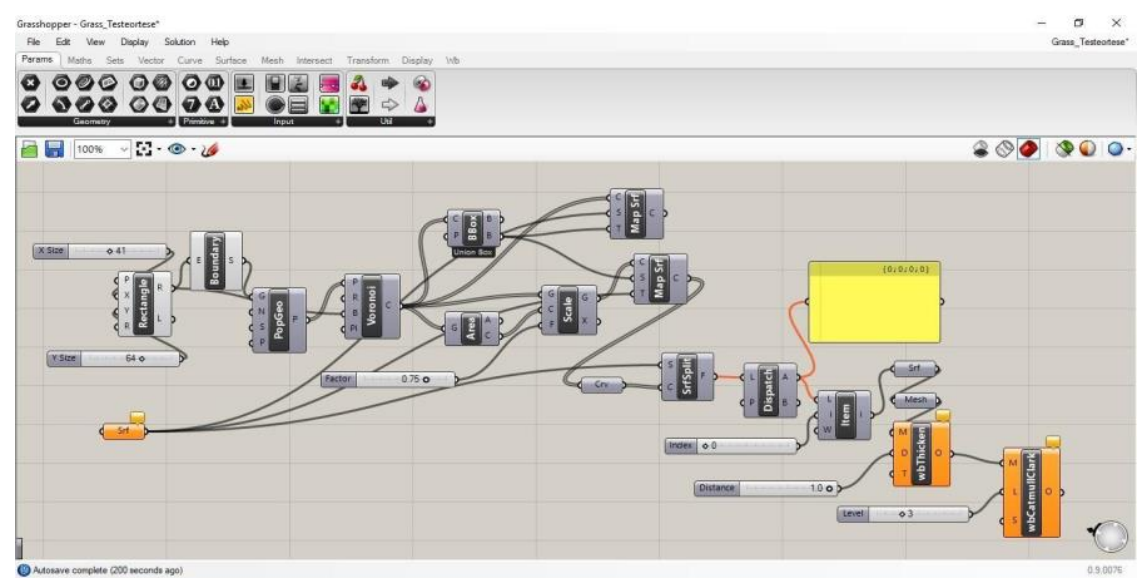

Após a montagem da nuvem de processos construtivos é necessário que esta seja vinculada ao retângulo que receberá as formas paramétricas. Para isso é preciso vincular a ferramenta Surface, caracterizada pela sigla Srf, com a superfície da região em que se pretende aplicar a parametrização, neste caso o retângulo.

Ao vincular a ferramenta da nuvem com o retângulo, este assume imediatamente a forma parametrizada definida no plug-in Grasshopper, possibilitando verificar a forma da parametrização que será utilizada futuramente na superfície da órtese. Através da nuvem gerada no Grasshopper também é possível alterar valores de ferramentas, modificando em tempo real formas do retângulo parametrizado para chegar à forma desejada, conforme visto na Figura 10.

Figura 10: Retângulo parametrizado

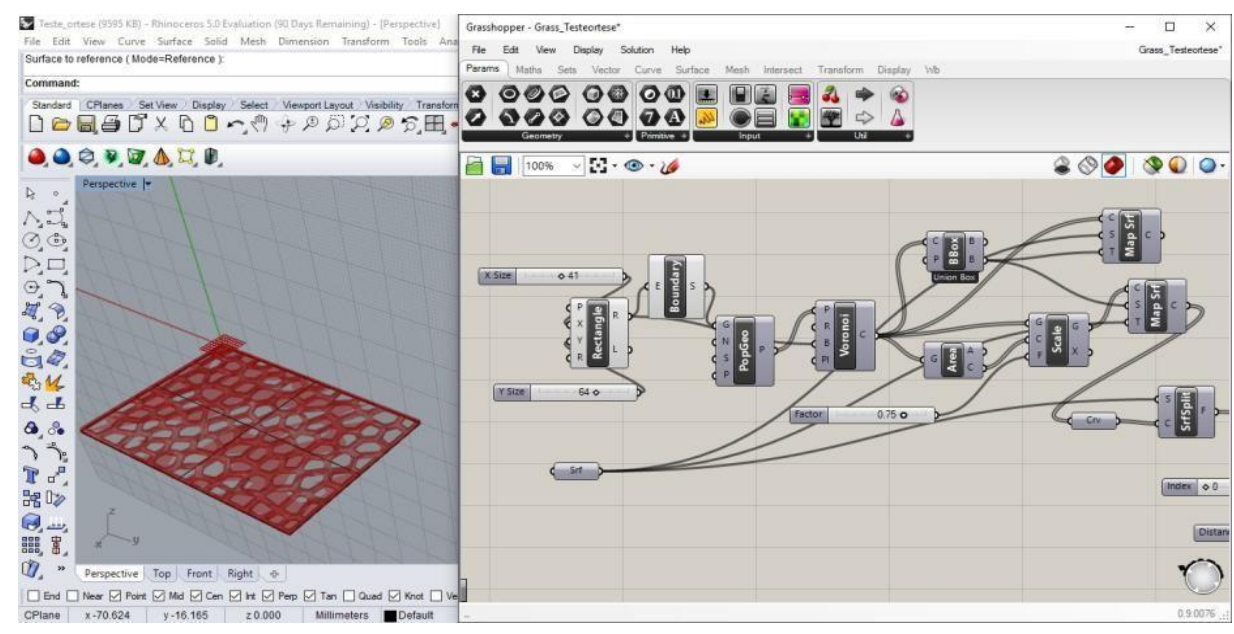

130 Congresso Brasileiro de Pesquisa e Desenvolvimento em Design, Univille, Joinville (SC) 
Mantendo a nuvem e o plug-in abertos, a malha base que contém informações ergonômicas do membro do paciente deve ser importada para utilização no Rhinoceros. Isso é feito através do comando File - Import.

Nesse caso a malha importada para o software Rhinoceros é a mesma fornecida por Catapan et al. (2016) e utilizada no estudo dos pontos críticos de tensão da órtese. Essa foi selecionada por conter a geometria da superfície do membro do paciente e também já existir a órtese em uso, para fins de comparações entre a atual e a proposta neste trabalho.

Figura 10: Malha importada no modelo

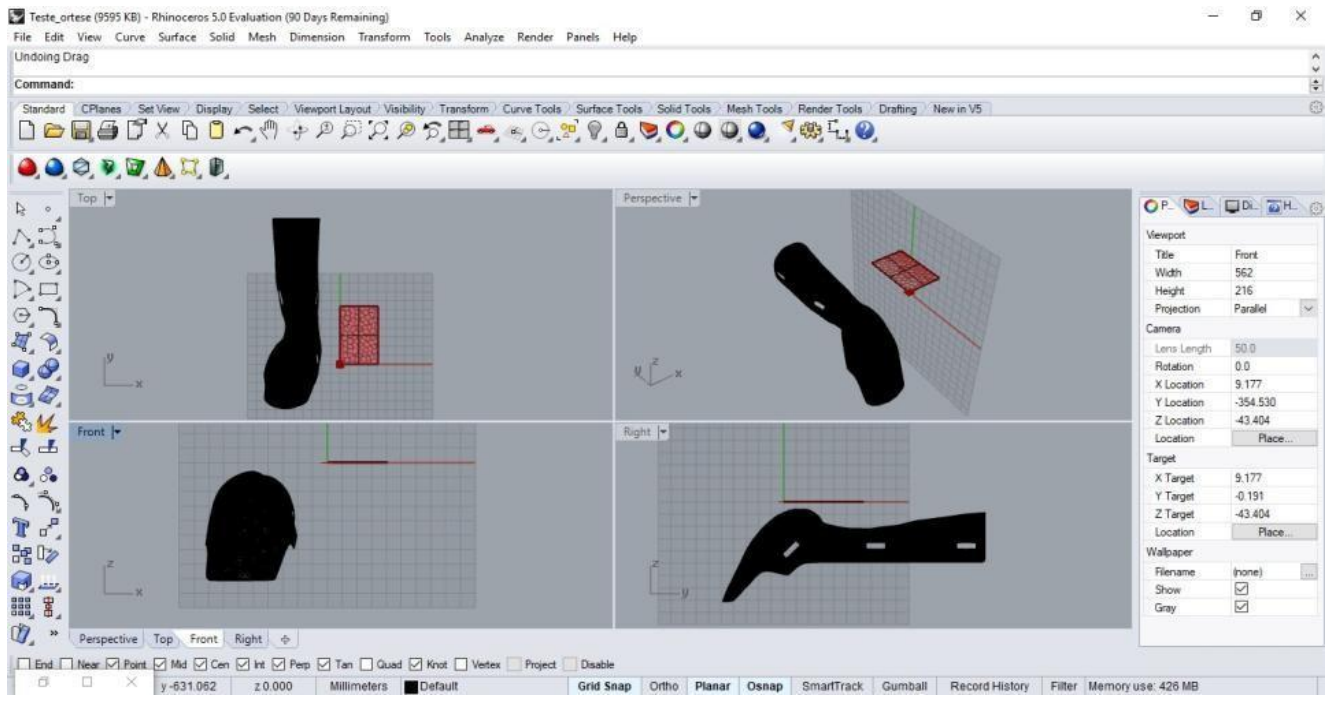

Posteriormente a importação da malha para o modelo e pensando nas modificações futuras de aproximação e afastamento dos pontos da estrutura parametrizada, são necessárias modificações na superfície retangular em que foram aplicados os parâmetros construtivos. Visto que esta estrutura é utilizada como a superfície da proposta final e buscando atender as conclusões apresentadas no presente trabalho, é necessário ativar e criar mais pontos de modificação no retângulo, preparando-o para ser aplicado na malha (Figura 11).

Figura 11: Alinhamento da malha com o retângulo parametrizado

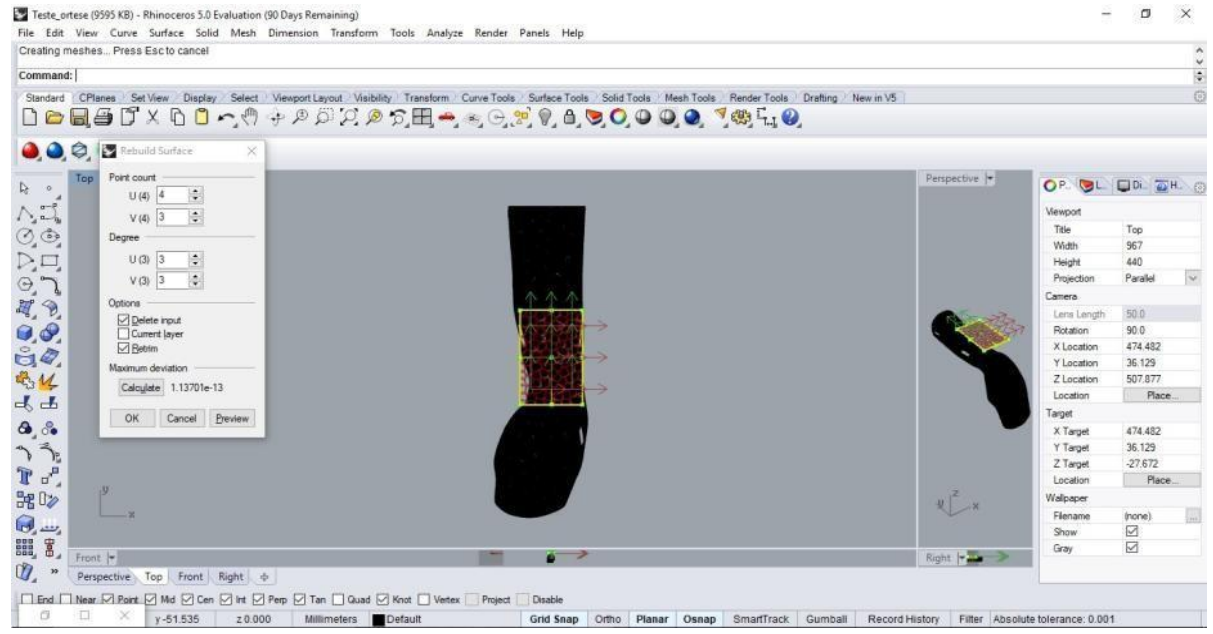


Para isto, como exemplificado na Figura 11, é aumentada a quantidade de pontos que dividem o retângulo - facilitando a edição da geometria da superfície após aplicação na malha. Feita a ativação dos pontos, a malha importada deve ser alinhada ao retângulo parametrizado, para que o mesmo seja modificado por vistas auxiliares de modo que este fique posicionado superior à malha, sobrepondo-a em relação à vista superior (Figura 12).

Figura 12: Retângulo e malha alinhados

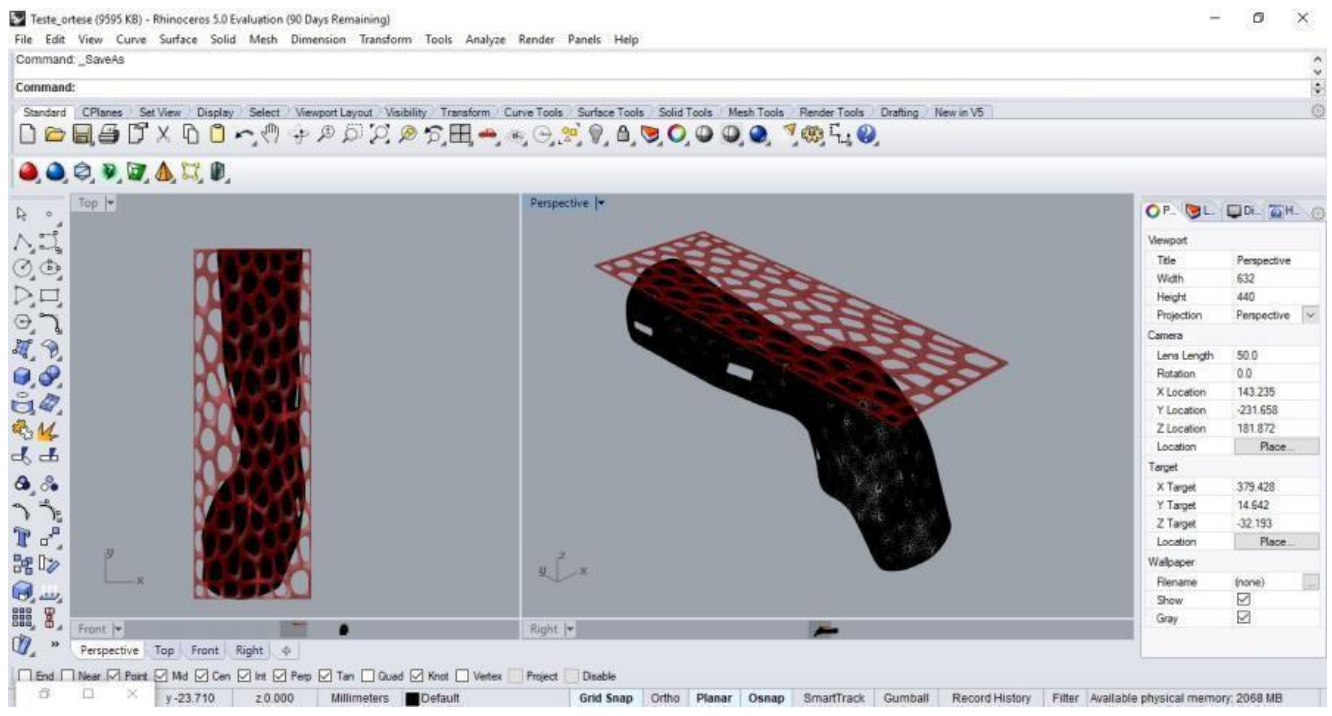

Finalizado o alinhamento, é possível começar a dar forma para a superfície parametrizada fazendo uso do próprio software Rhinoceros. Para isto a superfície do retângulo deve ser depositada sob a superfície externa da malha, anteriormente importada. Lembrando que a superfície da malha contém a geometria do membro do paciente, uma vez que a parte inferior da espessura do retângulo toma a forma da malha, é importante posicionar ambos de forma correta a fim de não prejudicar a forma interna do produto final. Feito isto, o retângulo parametrizado é moldado a possuir a mesma forma da malha que contém a geometria desejada.

Figura 13: Processamento da superfície parametrizada sobre a malha em formato da órtese

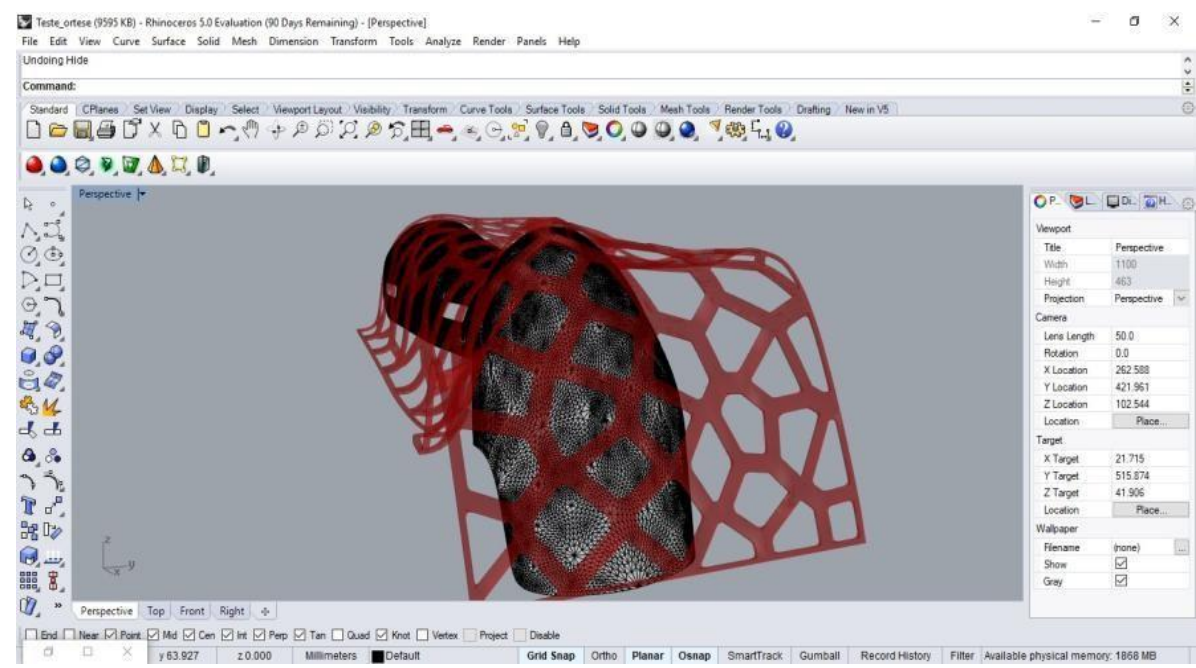


O processo de modelar a superfície parametrizada no formato da malha base é criando uma relação entre as duas superfícies, permitindo que o software processe a sobreposição de uma superfície sobre à outra. Enquanto o software processa a sobreposição é possível esconder a geometria primária do retângulo, facilitando a visualização do resultado da nova superfície, como registrado na Figura 13.

Após a superfície parametrizada tomar forma da órtese, o modelo já está pronto para a sua confecção como demonstrado na Figura 14.

Figura 14: Modelo finalizado - sem correção dos pontos críticos de tensão

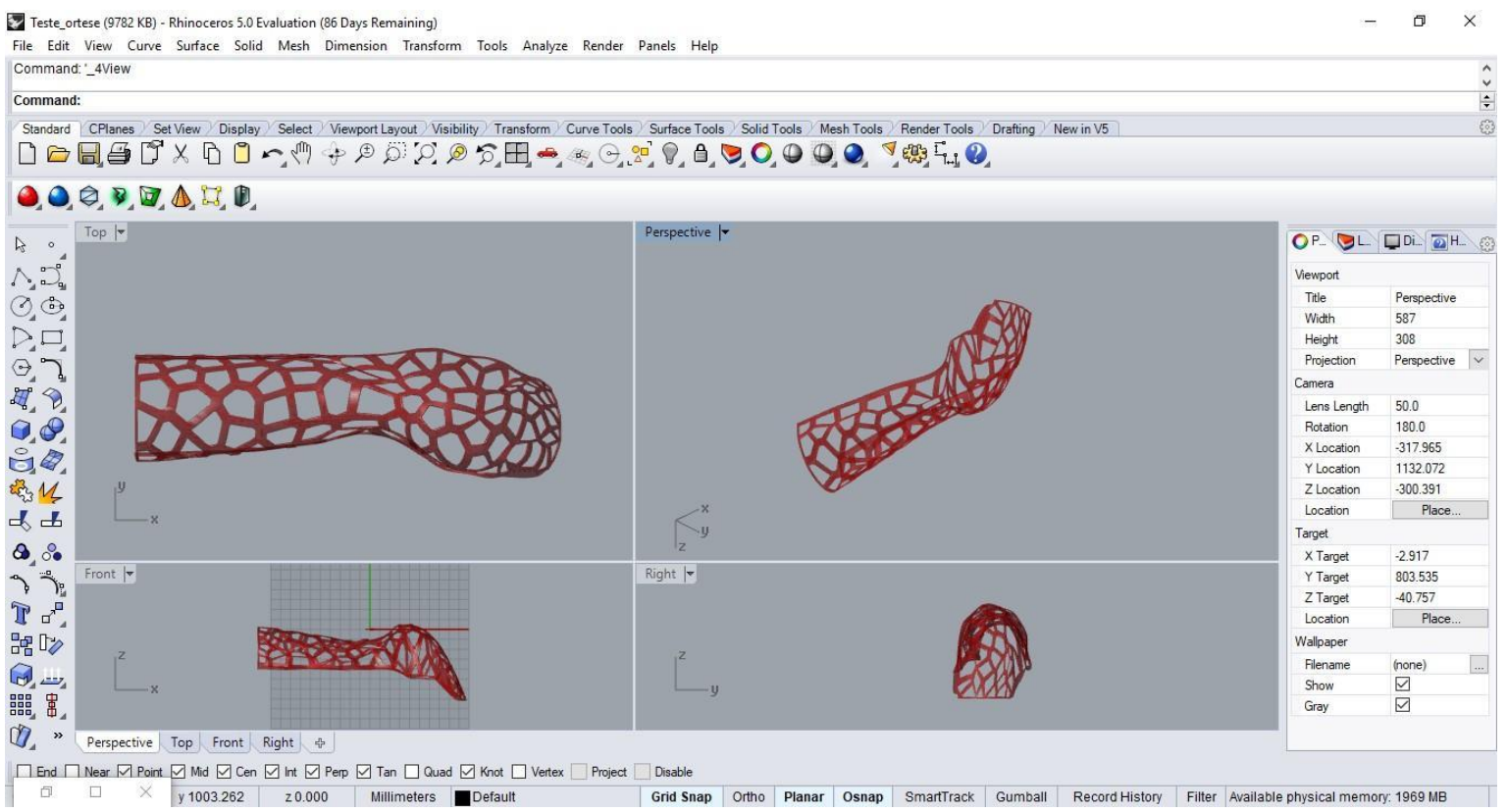

4.2.2 Proposta de resolução aos problemas de pontos críticos do modelo

Possuindo o protótipo virtual do modelo parametrizado, é possível trabalhar manualmente os pontos da superfície do mesmo, a fim de promover ajustes na geometria em busca de propor soluções aos problemas de vulnerabilidade desta. Para fazer os ajustes propostos anteriormente pelo profissional de saúde, é necessário habilitar os pontos base da superfície do retângulo, agora modificado, no software Rhinoceros.

A habilitação destes pontos é feita através do comando PointsON que deixa todos os pontos do modelo visíveis, gerando uma malha externa de NURBS (Non Uniform Rational Basis Spline) para facilitar a modelagem orgânica da nova superfície.

Através dos pontos habilitados é possível modificar o modelo através de modelagem manual, utilizando as quatro vistas para afastar ou aproximar pontos unicamente ou linhas de pontos. As modificações são promovidas com finalidade a deixar as regiões de vulnerabilidade da superfície mais compactadas aumentando a resistência da região, como exemplo na imagem abaixo, conforme apresentado na Figura 15. 
Figura 15: Modificações dos pontos vulneráveis

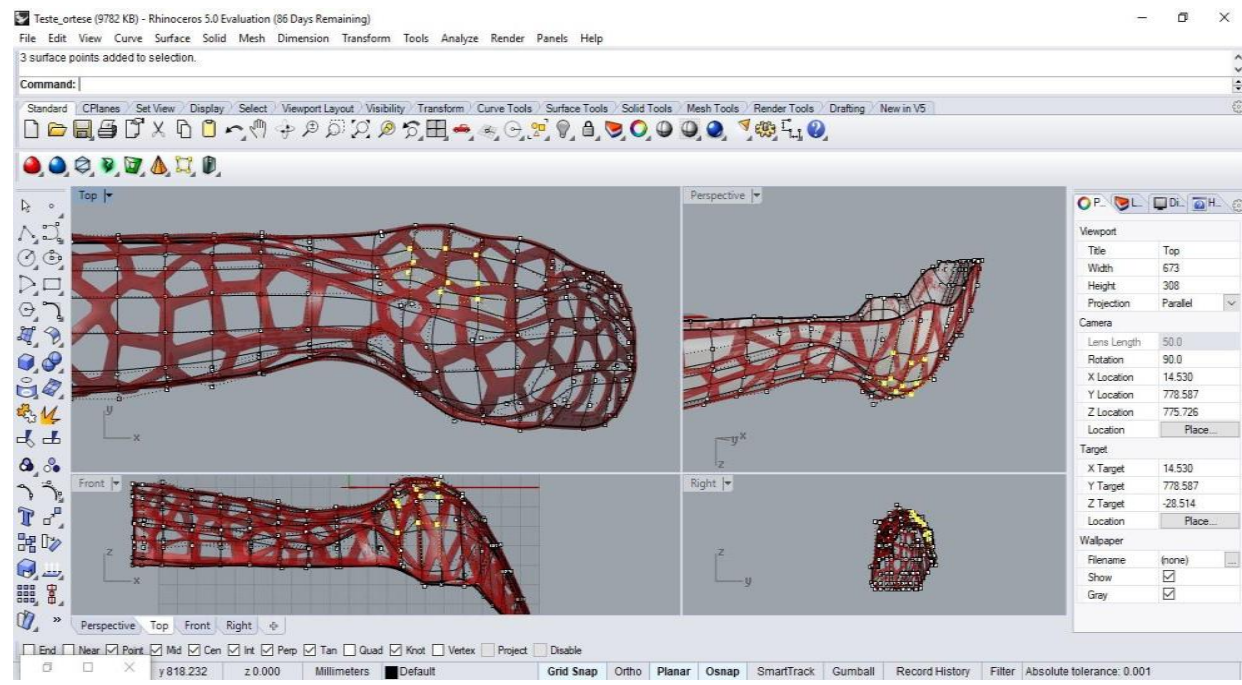

Outra solução sugerida a fim de solucionar a baixa resistência de pontos críticos da órtese é fazer uso de nervuras nas regiões em que a o material possa sofrer deformações. Como demonstrado na Figura 16, o uso de nervura seria recomendado com intuito de diminuir a quantidade de material e ser mais prático em relação ao processo de aumento da espessura da região - opinado pelo profissional de saúde.

Figura 16: Proposta para adição de nervura em pontos críticos

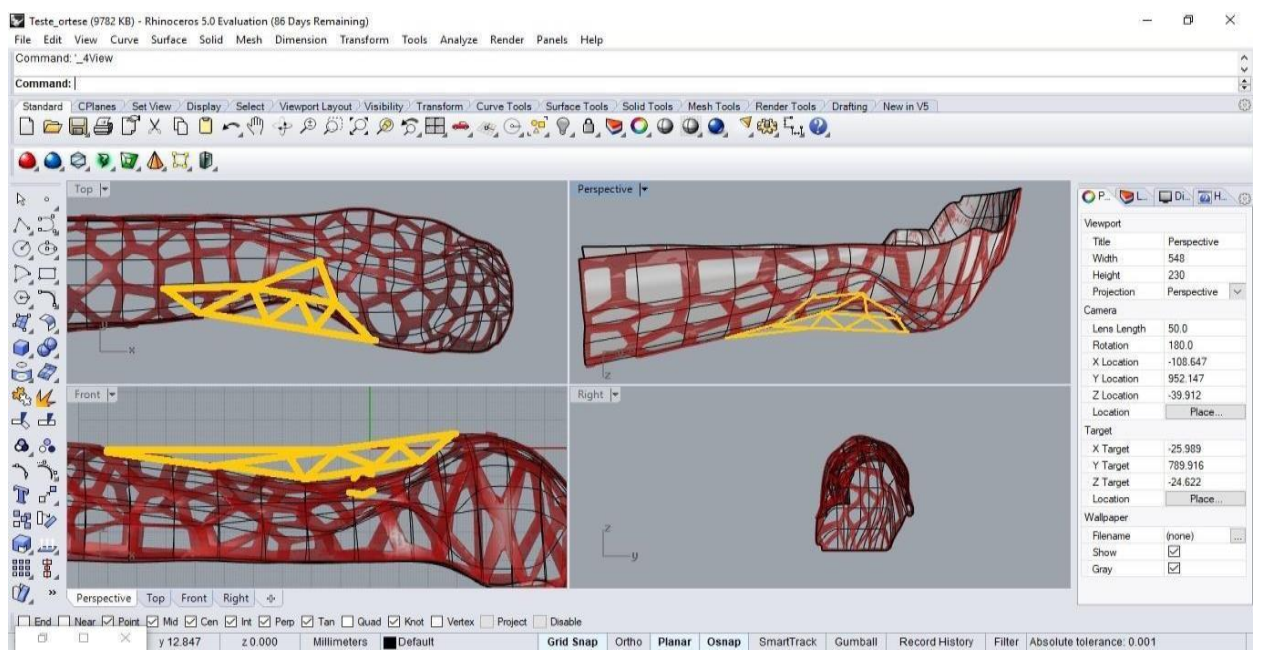

\section{CONCLUSÃO}

Com a finalização da proposta deste trabalho realizada, é possível indicar o uso da modelagem paramétrica no campo de desenvolvimento de produto para tecnologia assistiva.

O uso da parametrização demanda maior tempo para a fabricação do modelo se comparado 
à fabricação de protótipos já desenvolvidos sem o uso da técnica. Entretanto, o processo de modelagem apresentada atribui mais complexidade que a modelagem das órteses referenciadas nesta pesquisa.

Considerando que a fabricação de uma órtese estática de tecnologia assistiva é feita por necessidade de correção ou imobilização de um membro que deve ser posicionado de forma diferente à que se encontra. Segundo relato do profissional de saúde, que auxiliou no desenvolvimento desta pesquisa, quando a órtese tem como objetivo a correção do membro, é quase certo que esta irá sofrer com forças aplicadas sobre ela. Tais forças são resultantes da contração do membro do paciente, podendo comumente torcer, entortar e até quebrar o protótipo se mal planejado.

Além de fornecer uma boa estrutura para a imobilização do membro, o conceito também possui fatores relevantes: redução do uso de materiais, leveza, facilidade na higienização e customização - a serem implantados na fabricação de órteses para tecnologia assistiva.

Tornando possível o desenvolvimento de órteses customizada através de parametrização, a pesquisa conclui que este conceito pode ser implementado a área de tecnologia assistiva. Todavia, a mesma pode ser implantada na fabricação de tais produtos, mantendo as soluções já existentes em modelos anteriormente desenvolvidos.

Em relação a melhorias do conceito proposto, verifica-se a necessidade de estudos relacionados a reestruturar, com nervuras, o modelo proposto. Também, maneiras mais claras de pontos de tensões na órtese, dada o problema do usuário.

\section{Referências}

BARBOSA, W.; Araújo, A. L.; Celani, G. Modelagem paramétrica para o projeto e produção automatizados de uma peça de mobiliário: um exercício de aplicação. SIGraDi 2012, p. 561-565. Fortaleza, 2012.

BERSCH, R. Introdução à Tecnologia Assistiva. Porto Alegre, 2013. Disponível em: < http://www.assistiva.com.br/Introducao_Tecnologia_Assistiva.pdf > Acesso em: 28. Abr. 2016

CATAPAN, M. F.; FOSSILE FILHO, E.; ARAIUM, L. C.; DIETER, L.; OKIMOTO, M. L.; VILLAS BOAS, M. N.; SILVA, C.; STROBEL, C. Product Development of Assistive Technology for the Feet of a Person with Cerebral Palsy, 8th International Conference on Applied Human Factors and Ergonomics (AHFE 2017). Los Angeles - Califórina - USA, 2017.

CELANI, G. \& PUPO, R. Prototipagem Rápida e Fabricação Digital para Arquitetura e Construção: Definições e Estado da Arte no Brasil. Cadernos de Pós-Graduação em Arquitetura e Urbanismo. V.8, n.1, 2008.

COOK, A. M.; HUSSEY, S. M. Assistive Technologies: Principles and Practices. St. Louis, Missouri. Mosby - Year Book, Inc., 1995.

DEZEEN MAGAZINE. CORTEX 3D - Printed cast by jake evill. Jun.2013. Disponível em: <http://www.dezeen.com/2013/06/28/cortex-3d-printed-cast-forbone-fractures-jake-evill/> Acesso em: 08.Jun.2016.

EDELSTEIN, J. E.; BRUCKNER, J. Órteses - Abordagem Clínica. Série Physio - Fisioterapia Prática. Rio 
de Janeiro: Editora LAB, 2006.

FLORIO, W. Modelagem paramétrica, criatividade e projeto: duas experiências com estudantes de arquitetura. Gestão e Tecnologia de Projetos. V.6 - nํ2, p.43-66, 2011.

FOGGIATTO, J. S.; VOLPATO, N.; BONTORIN, A. C. B.; Recomendações para modelagem em sistemas CAD-3D. 4ํ COBEF. São Paulo, 2007.

FOGGIATTO, José A., POIER, Paloma H., FERNANDES, Bruno O. Uso da Impressão 3D na Fabricação de Órteses - Um Estudo de Caso. IDEMi 2015. Florianópolis, 2015.

GALVÃO FILHO, T. A. A. Tecnologia Assistiva: de que se trata? In: MACHADO, G. J. C.; SOBRAL, M. N. (Orgs.). Conexões: educação, comunicação, inclusão e interculturalidade. 1 ed. Porto Alegre: Redes Editora, p. 207-235, 2009.

GOIA, D. N. Estudo e projeto conceitual de órtese auto-articulada para correção de deformidade em desvio ulnar para portadores de artrite reumatóide. Dissertação de Mestrado. São Carlos: USP, 2012.

MEDEIROS, I. L.; PUPO, R.; KEGLER, A. J. M.; BRAVIANO, G. Prototipagem rápida e design de produto assistivo. 11요DDESIGN, 2014. Gramado, 2014.

PADRINI, P. F. Reabilitação da mão. São Paulo: Editora Atheneu, 2006.

SARTORETTO, M. L.; BERSCH, R. O que é tecnologia assistiva?. 2014. Disponível em: < http://www.assistiva.com.br/tassistiva.html > Acesso em: 28. Abr. 2016.

VILLAS BOAS, M. N.; BROGIN, B.; OKIMOTO, M.L.; CATAPAN, M. F.; FOGGIATO, J. A.; ROSENMANN, G. C.; WEIGERT, M. C. Fabricação de órtese de membro superior por meio de escaneamento tridimensional e manufatura aditiva. CONEM, 2016. Fortaleza, Agosto 2016.

VOLPATO, N, Et Al.. Prototipagem rápida: tecnologias e aplicações. São Paulo: Editora Edgard Blucher Ltda, 2007 